\title{
DEMANDA ENERGÉTICA NA PRODUÇÃO DE SORGO SILAGEIRO NA REGIÃO METROPOLITANA DO ESTADO DO RIO DE JANEIRO
}

\author{
Camila Madureira Duarte1, João Paulo Barreto Cunha², Marcus Vinicius Morais de Oliveira ${ }^{3} \&$ Livan Pereira Honorato ${ }^{4}$ \\ 1 - Bolsista FAPERJ, Graduanda do curso de Eng. Agrícola e Ambiental, UFRRJ, madureira.camila@gmail.com \\ 2 - Engenheiro Agrícola, Professor Adjunto, DE/UFRRJ, jpbcunha@ufrrj.br \\ 3 - Engenheiro Agrícola, Professor Adjunto, DE/UFRRJ, oliveiraufrrj@gmail.com \\ 4 - Bolsista CNPq/ PIBIC, Graduando do curso de Eng. Agrícola e Ambiental, UFRRJ, livanilson@hotmail.com
}

\section{Palavras-chave: alimentação animal análise energética balanço energético entradas e saídas}

\begin{abstract}
RESUMO
O balanço energético permite identificar as possíveis entradas e saídas de energia no processo de produção, resultando no saldo energético final do processo produtivo. O objetivo do trabalho foi avaliar os fluxos de energia e a demanda energética de dois sistemas de manejo do solo para a produção de sorgo silageiro na região metropolitana do estado do Rio de Janeiro. Foram realizados o estudo da eficiência energética e o balanço energético, quantificando o coeficiente energético de cada componente envolvido no processo de produção e determinando as matrizes de consumo energético nas formas de insumos, mão-de-obra, equipamentos, produção de silagem e restos culturais. Utilizou-se delineamento de blocos casualizados com cinco repetições, onde os tratamentos foram constituídos por sistema de preparo convencional do solo (SC) e sistema de preparo convencional com gradagem pré-plantio (SCG). Para os dados das entradas e saídas de energia, do balanço energético e da eficiência, os mesmos, quando significativos, foram submetidos ao teste de comparação de média Scot-Knott a $5 \%$ de probabilidade. Os resultados indicaram que a contribuição do dispêndio energético dos fertilizantes e combustíveis foi determinante para o elevado consumo energético dos sistemas de cultivo estudados, sendo o efeito contrário para as energias referentes às fontes de origem biológica. Para as condições do presente experimento, sob o ponto de vista energético, o sistema com gradagem adicional (SCG) apresentou-se energeticamente mais viável, apresentando maior produtividade e eficiência na conversão de energia.
\end{abstract}

\section{Keywords:}

animal feed

energy analysis

energy balance

inputs and outputs
ENERGY DEMAND IN THE PRODUCTION OF SILAGE SORGHUM IN THE METROPOLITAN REGION OF THE RIO DE JANEIRO STATE \begin{abstract}
The energy balance allows identifying the possible inputs and outputs of energy in the production process, resulting in the final energy balance of the production process. The aim of this work was to evaluate the energy flows and the energy demand of two soil management systems for the production of silage sorghum in the metropolitan region of the Rio de Janeiro state. The study of energy efficiency and energy balance was performed by quantifying the energy coefficient of each component involved in the production process and determining the energy consumption matrices in the forms of inputs, labor, equipment, silage production and cultural remains. A randomized block design with five replications was used, in which the treatments were constituted by conventional soil preparation system (SC) and conventional preparation system with tillage pre-planting (SCG).Energy input and output data, energy balance and efficiency were considered significant when applied to the Scot-Knott average comparison test at 5\% probability. The results indicated that the contribution of energy expenditure of the fertilizers and fuels was determinant for the high energy consumption of the cultivated systems studied. The opposite effect was obtained for the energies referring to the sources of biological origin. Considering the conditions of the present experiment, the system with additional tillage (SCG) was energetically more viable, presenting higher productivity and energy conversion efficiency.
\end{abstract}




\section{INTRODUÇÃO}

Analisar a sustentabilidade de qualquer sistema de produção agrícola não é somente com base na produção em relação ao custo/lucro e com base nas energias aplicadas ao sistema. De uma maneira geral, a agricultura moderna passou usar a natureza (energia solar, ar, água, nutrientes), como também a energia adicionada pelo homem na forma de fertilizantes, combustíveis e agroquímicos (SANTOS \& SIMON, 2010).

Dessa forma, a viabilidade dos sistemas se dá por meio da realização do balanço de energia, que se baseia no princípio físico de conservação de energia, também conhecido como primeiro princípio da termodinâmica, ou seja, a variação de energia em um processo pode ser explicada pelo balanço de energia, isto é, entradas e saídas de energia (VELOSO et al., 2012).

A realização do balanço energético visa principalmente estabelecer os fluxos de energia, identificando sua demanda total, a eficiência refletida pelo ganho de energia e a energia necessária para produzir ou processar uma unidade de determinado produto (BUENO et al., 2000). Para tal, diversas metodologias são utilizadas, permitindo que as energias empregadas no sistema sejam classificadas como direta e indireta. A energia direta utilizada em um processo produtivo não inclui somente o combustível fóssil utilizado, mas também outras formas de energia derivadas do petróleo, tais como aquelas contidas nos lubrificantes e nos adubos. Porém, um estudo completo da energia investida também deve levar em consideração as energias de origem biológica, como o trabalho humano e aquela contida nas sementes. A energia indireta utilizada na agricultura é aquela empregada através do uso de máquinas e implementos necessários à produção. Nesse processo, quantificam-se todos os insumos utilizados e produzidos que são transformados em unidade de energia, segundo o poder calorífico de cada um (RIQUETTI et al., 2012).

Pensando em alimentação animal, a principal fonte de volumoso é o pasto. Entretanto, em sistemas de confinamento, são utilizados alimentos conservados, e a disponibilidade de forragem no para os animais fica condicionada à produzir a forragem na época de seca ou realizar a retirada dos animais da região de pastejo (MONTANARI et al., 2013).

No setor agrícola do estado do Rio de Janeiro, basicamente boa parte da produção está baseada na pecuária de corte e leiteira. Uma das culturas exploradas para fins de silagem é o sorgo, apresentando maiores produtividades e menor tolerância às condições de déficit hídrico, além de representar boa alternativa de cultivo em regiões propensas a estiagem e de baixa fertilidade do solo (CIFUENTES et al., 2014; MONATANARI et al., 2015).

Adicionalmente, as características citadas vêm promovendo reduções dos custos de produção,daquelascultivadas solteiras ou em sistemas integrados para a produção de silagem (COSTA et al., 2015). Diante exposto, o presente trabalho teve como objetivo avaliar o balanço energético e a conversão energética na produção de sorgo forrageiro na região metropolitana do estado do Rio de Janeiro.

\section{MATERIAL E MÉTODOS}

O experimento foi conduzido em solo arenoso (EMBRAPA, 2013), em uma área de produção agrícola pertencente à Universidade Federal Rural do Rio de Janeiro (UFRRJ), localizada no campus Seropédica, no Rio de Janeiro, com coordenadas geográficas de $22^{\circ} 46^{\prime} 24^{\prime \prime} \mathrm{S}$ e $43^{\circ} 42^{\prime} 08^{\prime}$ ' W, com clima do tipo Aw, segundo a classificação de Köppen,provavelmente devido à proximidade do mar e a inexistência de uma estação seca rigorosa, visto que o Awrefere-se ao clima de savanas, que não é um tipo de vegetação da região.

Foi utilizado delineamento experimental em blocos casualizados (DBC), com cinco repetições. Para efeito de estudo, foram comparados dois tratamentos denominados de sistema convencional (SC), constituído de subsolagem, uma passada de grade pesada e uma passada grade leve; e sistema convencional com gradagem adicional (SCG), constituído por subsolagem, uma passada de grade pesada e duas passadas com grade leve, sendo a 
segunda realizada no período de pré-plantio.

Aeficiência energética $(\eta)$ dos sistemas foi obtida por meio da razão entre toda energia convertida e a consumida, com base nas quantidades estimadas de entrada e saída de energia, obtida durante o acompanhamento do ciclo de produção, conforme equação 1 .

$$
\eta=\frac{\text { Saidas energéticas }}{\text { entradas energéticas }}
$$

A energia específica (Es) tem sido amplamente utilizada para expressar a quantidade de energia investida para produzir uma quantidade unitária do produto (equação 2), enquanto a produtividade energética $(\mathrm{Pe})$ mede a quantidade de produto produzido por unidade de entrada de energia, sendo o inverso da energia específica.

$$
\begin{aligned}
& \text { Es }\left(M J K^{-1}\right)=\frac{\text { Entradas energéticas }}{\text { Produtividade }} \\
& P e=\left(k g \mathrm{MJ}^{-1}\right)=\frac{\text { Produtividade }}{\text { Entradas energéticas }}
\end{aligned}
$$

Visando realizar o balanço energético dos sistemas estudados, de maneira similar à metodologia adotada pelos autores Cunha et al. (2015) e Martins et al. (2015), se fez necessário relatar os componentes energéticos envolvidos nos diferentes sistemas de produção de sorgo estudados, como mostra o fluxo energético da Figura 1. Dessa maneira, como energia útil, foi considerada a produção da silagem colhida e a produção de restos culturais, servindo como material de cobertura para futuros cultivos.

Como energia direta, levou-se em consideraçãoe utilizou-se aquela referente aos combustíveis, lubrificantes e graxa, mão-de-obra, sementes, fertilizantes e defensivos, enquanto que para energia indireta foram considerados os gastos referentes às máquinas e implementos agrícolas (VELOSO et al., 2012).

De acordo com a metodologia descrita por Assenheimeret al. (2009), o balanço energético foi obtido mediante a transformação em unidades calóricas dos componentes de entrada envolvidos no sistema de produção de sorgo para silagem, seguindo-se os coeficientes energéticos estabelecidos por diversos autores, conforme estão apresentados na Tabela 1 .

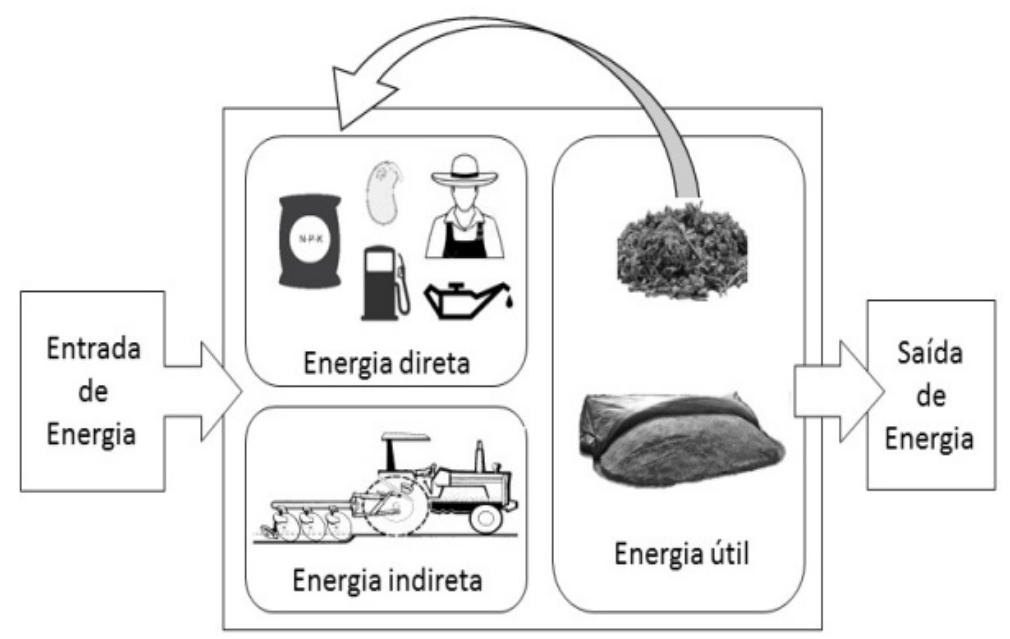

Legenda

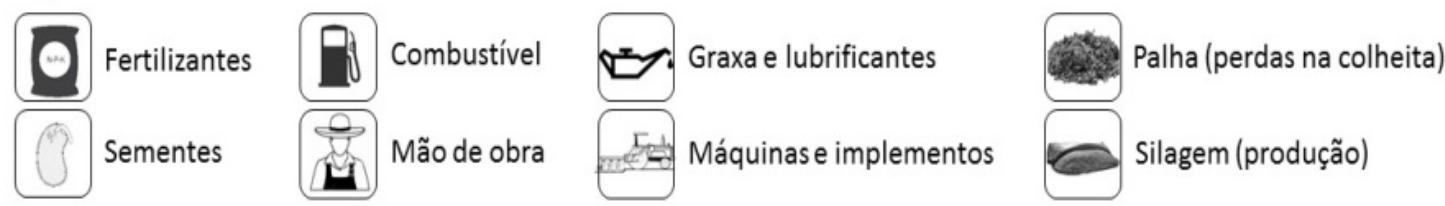

Figura 1. Fluxo energético nos sistemas de produção de sorgo para os diferentes tratamentos avaliados. 
Tabela 1. Componentes energéticos referentes ao sistema de produção de silagem de sorgo e seus respectivos coeficientes energéticos.

\begin{tabular}{lccc}
\hline Componentes de entrada & $\begin{array}{c}\text { Coeficiente } \\
\text { energético }\end{array}$ & Unidade & Fonte \\
\hline Mão de obra & 2,96 & $\mathrm{MJ} \mathrm{homem} \mathrm{h}^{-1}$ & Campos et al. (2009) \\
Sementes de sorgo & 59,54 & $\mathrm{MJ} \mathrm{ha}^{-1} \mathrm{~kg}^{-1}$ & Pimentel (1980) \\
Adubação (N) & 74,00 & $\mathrm{MJ} \mathrm{kg}^{-1}$ & Romanelli\& Milan (2005) \\
Adubação $\left(\mathrm{P}_{2} \mathrm{O}_{5}\right)$ & 12,56 & $\mathrm{MJ} \mathrm{kg}^{-1}$ & Romanelli\& Milan (2005) \\
Adubação $\left(\mathrm{K}_{2} 0\right)$ & 6,70 & $\mathrm{MJ} \mathrm{kg}^{-1}$ & Romanelli\& Milan (2005) \\
Calcário & 0,23 & $\mathrm{MJ} \mathrm{kg}^{-1}$ & Macedônio \&Picchioni (1985) \\
Óleo Diesel & 43,71 & $\mathrm{MJ} \mathrm{L}^{-1}$ & Bueno (2002) \\
Óleo Lubrificante & 39,43 & $\mathrm{MJ} \mathrm{L}^{-1}$ & Campos et al. (2004) \\
Graxa & 43,38 & $\mathrm{MJ} \mathrm{kg}^{-1}$ & Campos et al. (2004) \\
Trator & 69,83 & $\mathrm{MJ} \mathrm{kg}^{-1}$ & Zaniniet al. (2003) \\
Implementos & 57,20 & $\mathrm{MJ} \mathrm{kg}^{-1}$ & Assenheimeret al. (2009) \\
Silagem sorgo (baixo tanino) & 18,98 & $\mathrm{MJ} \mathrm{kg}^{-1}$ & Furlan et al. (2006) \\
\hline
\end{tabular}

Os gastos energéticos com a mão de obra utilizada foram obtidos em função da quantidade de horas e pessoas demandadas para a operação, multiplicadas pelo coeficiente energético referente a este fator.

A energia consumida por máquinas e equipamentos consistiu na aplicação do método baseado na depreciação energética descrito por Riquettiet al. (2012). Tal metodologia consiste em depreciar as máquinas e os implementos durante sua vida útil em função de suas massas. Dessa forma, para execução dos cálculos, empregou-se a Equação 4:

$$
D E E=\frac{(M \cdot C E) \cdot T U}{V U}
$$

em que,

$\mathrm{DEE}=$ demanda específica de energia, em MJ;

$\mathrm{M}=$ peso de máquinas ou equipamentos, em $\mathrm{kg}$;

$\mathrm{CE}=$ coeficiente energético de máquinas ou equipamentos, em $\mathrm{MJ} \mathrm{kg}^{-1}$;

$\mathrm{VU}=$ vida útil, em h; e

$\mathrm{TU}=$ tempo de uso, em $\mathrm{h}$.

Dessa maneira, computou-se o número de vezes da execução de cada tarefa e o tempo gasto em cada operação (hora máquina), enquanto a massa das máquinas e implementos foram obtidos junto aos catálogos de fabricantes. O consumo de combustível das operações mecanizadas realizadas foi obtido por meio das cadernetas de campo e posteriormente conferidos por meio da normativa ASAE D497.7 (ASABE, 2011), que em seguida foi multiplicado pelo seu respectivo coeficiente energético, no qual, juntamente com os gastos com graxa e lubrificantes, permitiu a obtenção de toda energia fóssil consumida.

Como fonte de tração, utilizou-se um trator agrícola MasseyFergunson MF4275, motor ciclo Diesel, potência nominal segundo ISSO 1585 de $55,1 \mathrm{~kW}(75 \mathrm{cv})$, tração dianteira auxiliar, rodados equipados com pneus diagonais dianteiros (12.4-24) e traseiros (16.9-30). Para a realização do manejo convencional de ambos os sistemas avaliados, utilizou-se um subsolador IMASA com três hastes, largura útil de $1240 \mathrm{kgf}$ e massa de 358kgf; grade pesada TATU Marchesan, modelo ATCR, dotadas com 14 discos, largura útil de $1500 \mathrm{~mm}$ e massa de $1531 \mathrm{kgf}$; grade niveladora KLR, modelo GN195, dotadas com 24 discos de 20", largura útil de $2145 \mathrm{~mm}$ e massa de $712 \mathrm{kgf}$.

Para a melhoria e correção do solo, foi realizada a calagem, onde se utilizou um distribuidor de calcário tipo cocho da marca Santa Izabel, previamente regulado para a dosagem de $350 \mathrm{~kg}$ por hectare, com largura de distribuição de $2200 \mathrm{~mm}$, 
massa de $295 \mathrm{~kg}$ e capacidade de carga de $550 \mathrm{~kg}$.

No processo de instalação da cultura do sorgo, foi utilizada uma semeadora-adubadora da marca TATU Marchesan, modelo $\mathrm{STP}^{2}$ com quatro módulos de semeadura espaçados em $800 \mathrm{~mm}$, onde a mesma foi previamente regulada para a aplicação de $286 \mathrm{~kg}$ de adubo NPK (4-14-8) e 121000 plantas por hectare.

As sementes de sorgo utilizadas para semeadura foram do tipo híbrido SHS570 (Santa Helena), caracterizadas por terem fins de silagem, serem resistentes às principais doenças da cultura, terem ciclo precoce enão terem tanino. Para o cálculo da energia fornecida pelas sementes, levou-se em consideração a quantidade de sementes utilizadas por hectare (144.058 unidades) e o peso de 1000 sementes $(0,159 \mathrm{~kg})$, determinado de acordo com a metodologia proposta pela Regra de Análises de Sementes (BRASIL, 2009).

No cultivo pós emergência, não foi realizada a aplicação de agroquímicos para a realização de controles fitossanitários. Desta forma, houve somente a realização de adubação de cobertura, onde foi acoplada a semeadora-adubadora STP $^{2}$ TATU Marchesan com quatro módulos de semeadura. Entretanto, dessa vez foi feita uma adaptação para liberar somente o fertilizante, com dosagem de 400kg de adubo NPK (4-14-8) por hectare.

A colheita do sorgo no estágio de forragem foi realizada com uma colhedora de forragens da marca Pinheiro, modelo Max 10, com uma linha de colheita acionada a 540rpm da TDP, bica giratória, rolo dotado com dez facas e massa de $552 \mathrm{kgf}$.

Concomitante ao processo de colheita, foi realizado o transporte do material colhido por meio de um trator da marca John Deere, modelo 5403, com motor ciclo Diesel e potência nominal de $48 \mathrm{~kW}$ $(65 \mathrm{cv})$, tração dianteira auxiliar, pneus diagonais dianteiros (12.4-24) e traseiros (16.9-28-8PR),que serviu como fonte de potência para tracionar uma carreta Triton, modelo TR791 com eixo simples, massas de $365 \mathrm{~kg}$ e capacidade de carga de $3000 \mathrm{~kg}$.

Para determinar a produtividade, foram colocados sacos de 200L na saída da colhedora e contabilizado o tempo para enchê-los, à velocidade média da operação. Com relação à quantidade de cobertura vegetal, utilizou-se um gabarito de
$1 \mathrm{~m}^{2}$, onde todo material vegetal remanescente foi recolhido manualmente, ensacado e pesado, permitindo determinar as saídas energéticas de cada sistema avaliado. Posteriormente, foi realizada a determinação da quantidade de matéria seca do material colhido por meio do método convencional em estufa com ventilação forçada, obtendo o valor de $37,3 \%$ do peso fresco total produzido.

Com posse dos dados obtidos para as entradas e saídas energéticas, energia líquida e eficiência energética de cada sistema de cultivo avaliado,eles foram submetidos à análise de variância, onde, quando significativos, foram submetidos ao teste de Scott-Knott a 5\% de probabilidade.

\section{RESULTADOS E DISCUSSÃO}

Os valores médios da produtividade e a cobertura vegetal estão apresentados na Tabela 2. Para efeito de comparação, foram relatados os valores para ambos os sistemas estudados, possibilitando assim verificar possíveis diferenças na condução da lavoura, sendo expressos em matéria seca. Estatisticamente, o sistema em que foi realizada a gradagem adicional (SCG) apresentou maior produtividade em relação ao outro sistema. A maior produtividade no sistema com a adoção gradagem adicional explica-se devido à alteração da estrutura do solo. Consequentemente, os agregados do solo foram reduzidos, visto que essa prática promove o corte e enterrio de invasoras, auxiliando o controle de pragas e patógenos no solo, além de facilitar o crescimento das plantas (SILVA et al., 2011).

Tabela 2.Valores médios das saídas energéticas em função dos sistemas de produção.

\begin{tabular}{lcc}
\cline { 2 - 3 } & \multicolumn{2}{c}{ Sistema de Manejo } \\
\hline & $\mathrm{SG}$ & SGC \\
\hline Silagem $\left(\mathrm{kg} \mathrm{ha}^{-1}\right)$ & $10966,2 \mathrm{~b}$ & $12868,5 \mathrm{a}$ \\
Restos culturais $\left(\mathrm{kg} \mathrm{ha}^{-1}\right)$ & $2731,7 \mathrm{a}$ & $3205,5 \mathrm{a}$ \\
\hline
\end{tabular}

Médias seguidas da mesma letra na linha não diferiram estatisticamente pelo teste Scott-knott a 5\% de significância.

Com relação à quantidade de cobertura vegetal, a mesma não apresentou diferença significativa para ambos os sistemas. De maneira geral, a cobertura vegetal é de suma importância não só sob 
o aspecto energético, mas principalmente para os cultivos subsequentes, uma vez que quanto maior a cobertura da superfície do solo, maior a proteção da estrutura do solo contra perturbações naturais e antropogênicas (BLANCO-CANQUI \& LAL, 2009).

As produtividades médias de matéria seca obtidas foram próximas à média dada pela fabricante para a mesma época do ano, ou seja, $13 \mathrm{t} \mathrm{ha} \mathrm{A}^{-1}$. O desempenho da maioria dos genótipos de sorgo silageiro é superior ao do milho, com resultados variando de 6,9 a $19,9 \mathrm{t} \mathrm{ha}^{-1}$ (CHIELLE et al., 2013).

O consumo médio diário de silagem de sorgo por um bovino é de $6,53 \mathrm{~kg} \mathrm{dia}^{-1}$ de matéria seca (SILVA et al., 2006). Desta forma, em um mês, a produtividade encontrada em um hectare do Sistema de Manejo Convencional (SC) seria capaz de alimentar 56 animais. Sob o mesmo ponto de vista, o sistema convencional com gradagem adicional (SCG) alimentaria aproximadamente 65 animais no mesmo tempo.

As energias consumidas e convertidas, obtidas para cada sistema de manejo, estão descritas na Tabela 3. De acordo com os resultados apresentados, foi possível verificar que,em ambos os sistemas avaliados, o uso de energia relativa aos fertilizantes utilizados na calagem, adubação de fundação e de cobertura e sementes de sorgo são sempre as mesmas, visto que a dosagem de aplicaçãodesses insumos, em $\mathrm{kg} \mathrm{ha}^{-1}$, foi a mesma em ambos os tratamentos.

A utilização da energia proveniente da mãode-obra, mesmo sendo uma pequena parcela na energia consumida, permite avaliar que o sistema com gradagem adicional apresentou valor maior em comparação ao sistema convencional, devido ao grau de intensidade da mecanização na cultura, ou seja, a quantidade de energia desse fator está diretamente relacionada ao número de operações realizadas para cada sistema.

Com relação ao balanço de energia, a Tabela 4 mostra que o sistema de manejo convencional (SC) utilizou a menor quantidade de energia (10528,6 $\left.\mathrm{MJ} \mathrm{ha}^{-1}\right)$, mesmo não diferindo estatisticamente do sistema com gradagem adicional (11128,3 $\left.\mathrm{MJ} \mathrm{ha}^{-1}\right)$. O resultado se dá justamente pela não realização da gradagem adicional pré-emergente, fato explicado pela menor utilização de máquinas e implementos.

Diversos autores têm verificado os gastos energéticos para diferentes culturas e em diferentes regiões. Quando comparada a energia de entrada dos sistemas de cultivo estudados com os resultados obtidos em outros países, é possível observar uma quantidade menor do que a utilizada porKalbande\& Mor (2008) na produção de sorgo.Para produzir mecanicamente sorgo na Índia, necessitaram de 12959,5 $\mathrm{MJ} \mathrm{ha}^{-1}$.

Tabela 3.Valores obtidos para as entradas e saídas energéticas (MJ) dos sistemas de manejo avaliados.

\begin{tabular}{lcc}
\hline Descrição energia $\left(\mathrm{MJ} \mathrm{ha}^{-1}\right)$ & $\mathrm{SC}$ & $\mathrm{SGC}$ \\
\hline Energia Direta & & \\
Mão-de-obra & 108,50 & 110,5 \\
Sementes & 1363,70 & 1363,7 \\
Óleo Diesel & 4206,9 & 4323,3 \\
Lubrificantes & 54,26 & 55,32 \\
Graxa & 13,96 & 14,42 \\
Fertilizantes & 3684,70 & 3684,70 \\
\hline Energia Indireta & & 822,10 \\
Trator & 650,84 & 754,34 \\
Implementos & 445,81 & \\
& & \\
\hline Saídas de Energia & & 59443,22 \\
Restos culturais & 53383,62 & 245553,75 \\
Produção & 209491,80 & \\
\hline
\end{tabular}


Ao analisar as saídas energéticas, é possível afirmar que o sistema com gradagem adicional (SCG) apresentou valores $13,8 \%$ superiores ao sistema convencional (SC). Diante do exposto, é possível afirmar que mesmo não havendo diferença entre as entradas, a saída energética teve um aumento por estar relacionada à mobilização do solo,devido agradagem adicional resultar em um faturamento dos agregados e o desenvolvimento de poros, facilitando assim ao desenvolvimento do sistema radicular e absorção de nutrientes.

Os valores de eficiência energética, energia específica e produtividade energética apresentada mostram que o sistema com gradagem adicional (SCG) foi superior em relação ao sistema convencional (SC). Tal resultado já era esperado, visto que a eficiência está diretamente relacionada com a produtividade do sistema e as saídas energéticas, ocasionando ganho de aproximadamente $9 \%$.

A quantidade de energia empregada no sistema de produção de sorgo é uma das grandes vantagens da cultura, seja para a produção de biomassa como em comparação ao milho para a produção de silagem ou grãos, pois trata-se de uma cultura energeticamente mais eficiente (REN et al., 2012).

Consequentemente, à medida que são obtidas maiores eficiências energéticas, há uma menor energia específica gasta e maior produtividade energética. Tal comportamento foi semelhante quando realizado o balanço de energia do sorgo em comparação com outras culturas, como Sefeedpariet al. (2013) avaliando sistemas de produção de silagem de milho.

Conforme a Figura 2, é possível observar um maior consumo da energia direta sobre a indireta, o que corrobora com os resultados obtidos por Kheyri\&Dahab (2016) no cultivo de sorgo. É possível observar a grande dependência de energia direta, principalmente as referentes ao uso de fertilizantes (média de 50,4\%), seguido da energia representada pelos combustíveis, lubrificantes e graxas (média de 34,6\%).

Tabela 4. Balanço energético, eficiência energética $(\eta)$, energia específica (Es) e produtividade energética (Pe) dos sistemas avaliados.

\begin{tabular}{ccccccc}
\hline Sistemas & $\begin{array}{c}\text { Entrada } \\
\left(\mathrm{MJ} \mathrm{ha}^{-1}\right)\end{array}$ & $\begin{array}{c}\text { Saída } \\
\left(\mathrm{MJ} \mathrm{ha}^{-1}\right)\end{array}$ & $\begin{array}{c}\text { Energia } \\
\text { Líquida }\left(\mathrm{MJ} \mathrm{ha}^{-1}\right)\end{array}$ & $\eta$ & Es & Pe \\
\hline SC & $10528,60 \mathrm{a}$ & $262875,42 \mathrm{a}$ & $252346,82 \mathrm{a}$ & $24,97 \mathrm{a}$ & $0,96 \mathrm{a}$ & $1,04 \mathrm{a}$ \\
SGC & $11128,30 \mathrm{a}$ & $304996,97 \mathrm{~b}$ & $293868,67 \mathrm{~b}$ & $27,41 \mathrm{~b}$ & $0,86 \mathrm{~b}$ & $1,16 \mathrm{~b}$ \\
\hline
\end{tabular}

Médias seguidas da mesma letra na coluna não diferiram estatisticamente pelo teste Tukey a 5\% de significância.

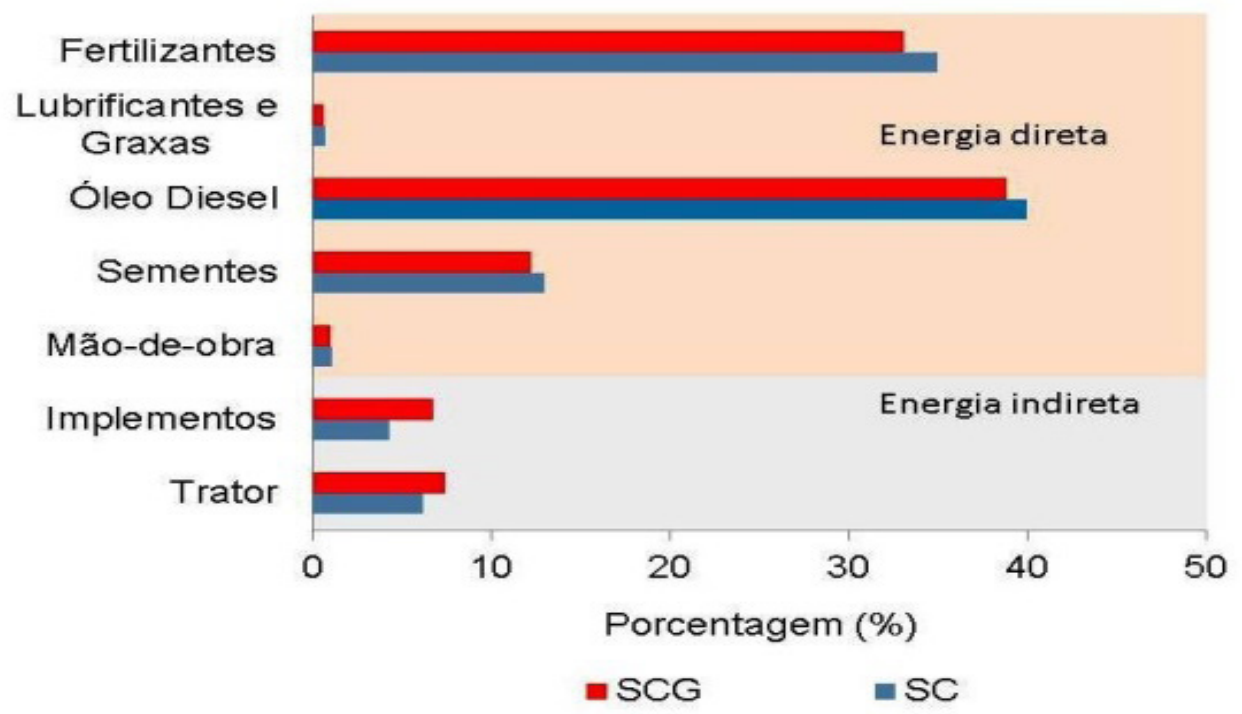

Figura 2. Participação das rubricas energéticas nas energias indiretas e diretas para a produção de sorgo. 
Atualmente, sistemas agrícolassão fortemente dependentes dos recursos fertilizantes, pesticidas e maquinário. No entanto, segundo Woods et al. (2010) não há uma relação linearentre entradas de energia e rendimento, ou seja, baixas entradas energéticas podem levar a rendimentos mais baixos e perversamente para maiores demandas de energia por tonelada de produto colhido.

O comportamento apresentado é semelhante em outras culturas silageiras, como o milho. Avaliando as entradas e saídas energéticas na produção de milho, Lozardehet al. (2011) verificaram gastos energéticos referentes ao uso de fertilizantes e combustíveis de até $76 \%$ da totalidade do dispêndio energético, enquanto os referentes à energia de origem biológica (sementes e mão-de-obra) foram pouco representativo, resultados semelhantes ao presente estudo.

Diversos autores citam o grande potencial da cultura do sorgo não somente para fins de silagem. Diante dos resultados, é possível afirmar o grande potencial de exploração da cultura por parte dos produtores da região metropolitana do estado do Rio de Janeiro, visto que, em diferentes regiões do planeta, o sorgo é consumido como fonte proteica na alimentação humana, para a produção de biocombustíveis e de biomassa para geração de energia elétrica, como também na recuperação de áreas degradadas (TAPHEE et al., 2015; LIU et al., 2015; SERRA et al., 2017).

\section{CONCLUSÕES}

- A contribuição do dispêndio energético dos fertilizantes e combustíveis foi determinante para o elevado consumo energético dos sistemas de cultivo estudados, havendo pouca contribuição da energia de fontes biológicas.

- O balanço energético mostra que o sistema de manejo com gradagem adicional (SCG) no pré-plantio mostrou-se energeticamente mais eficiente por promover maior mobilização do solo e consequente produtividade.

- Verifica-se um balanço energético positivo nas condições da região metropolitana do estado do Rio de Janeiro para ambos os sistemas avaliados.

\section{REFERÊNCIAS BIBLIOGRÁFICAS}

ASABE.Agricultural machinery management data ASAE D497.7.ASABE Standards.St. Joseph, 2011. 8p.

ASSENHEIMER, A.; CAMPOS, A.T.; GONÇALVES JÚNIOR, A.F.C. Análise energética de sistemas de produção de soja convencional e orgânica. Revista Ambiência, Guarapuava, v.5, p.443-455, 2009.

BLANCO-CANQUI, H.; LAL, R. Crop residue removal impactson soil productivity and environmental quality. CriticalReviews in Plant Science, v.28, p.39-163, 2009.

BUENO, O.C.; CAMPOS, A.T.; CAMPOS, A.T. Balanço de energia e contabilização da radiação global: simulação e comparativo. Buenos Aires: Faculdade de Agronomia, 2000.

BUENO, O.C. Análise energética e eficiência cultural do milho em assentamento rural, Itaberá/SP. Tese de Doutorado. Universidade Estadual Paulista de Botucatu, 2002, 147p.

BRASIL. Regras para análise de sementes. Secretaria de Defesa Agropecuária. - Brasília: Mapa/ACS, 2009. 399p.

CAMPOS, A.T.; SAGLIETTI, J.R.C.; BUENO, O.C.; CAMPOS, A.T.; KLOSOWSKI, E.S.; GASPARINO, E. Balanço energético na produção de feno de alfafa em sistema intensivo de produção de leite. Ciência Rural, Santa Maria, v.34, p.245251, 2004.

CAMPOS, A.T.; KLOSOWSKI, E.S.; SOUZA, C.V.; ZANINI, A.; PRESTES, T.M.V. Análise energética da produção de soja em sistema de plantio direto. Global Science Technology, Rio Verde, v.2, p.38-44, 2009.

CIFUENTES, R.; BRESSANI, R.; ROLZ, C.The potential of sweet sorghum as a source of ethanol and protein. Energy for Sustainable Development, v.21 p.13-19, 2014. 
COSTA, N.R.; ANDREOTTI, M.; BERGAMASCHINE, A.F.; LOPES, K.S.M.; LIMA, A.E.S. Custo da produção de silagens em sistemas de integração lavoura-pecuária sob plantio direto. Revista Ceres, Viçosa, v.62, n.1, p.9-19, 2015.

CHIELLE. Z.G.; GOMES. J.F.; ZUCHI. J.; GABE. N.L.; RODRIGUES. L.R. Desempenho de genótipos de sorgo silageiro no Rio Grande do Sul na safra 2011/2012. Revista Brasileira de Milho e Sorgo, Sete Lagoas, v.12. n.3. p.260-269, 2013.

CUNHA, J.P.B.; CAMPOS, A.T.; MARTINS, F.G.L.; PAULA, V.R.; VOLPATO, C.E.S.; SILVA, F.C. Demanda energética de diferentes manejos de solo no cultivo de milho. Bioscience Journal, Uberlândia, v.31, p.808-817. 2015.

EMBRAPA. Centro Nacional de Pesquisa de Solos. Sistema Brasileiro de Classificação de Solos. 3ed. Rio de Janeiro: Embrapa Solos, 2013. 353p.

FURLAN, A.C.; SCAPINELLO, C.; MOREIRA, I.; MARTINS, E.N. Avaliação nutricional da silagem de grãos úmidos de sorgo de baixo ou de alto conteúdo de tanino para coelhos em crescimento. Revista Brasileira de Zootecnia, Viçosa, v.35, p.775-784, 2006.

KALBANDE, S.R.; MORE, G.R. Assessment of Energy Requirement for Cultivation of Kharif and Rabi Sorghum. Karnataka Journal of Agricultural Science, Dharwad v.21, n.3, p.416420, 2008.

KHEIRY, A.N.O.; DAHAB, M.H. Energy Inputoutput analysis for production of selected crops in the central clay vertisols of Gezira agricultural scheme (Sudan).International Journal of Science and Research, Raipur, v.5, n.3, p.1215-1220, 2016.

LIU, H.; REN, L.; SPIERTZ, H.; YONGBIN, Z.; GUANG, H. An economic analysis of sweet sorghum cultivation for ethanol production in North China. GCB Bioenergy, n.7, p.1176-1184, 2015.
LORZADEH, S.H.; MAHDAVIDAMGHANI, A.; ENAYATGHOLIZADEH, M.R.; YOUSEFI, A. Energy Input-Output Analysis for Maize Production Systems in Shooshtar, Iran. Advances in Environmental Biology, v.11, n.5, p.36413644, 2011.

MACEDÔNIO, A.C.; PICCHIONI, S.A. Metodologia para o cálculo do consumo de energia fóssil no processo de produção agropecuária. Curitiba: Secretaria de Estado da Agricultura, 1985, v.1, 95p.

MARTINS, F.G.L.; BARBOSA, J.A.; CARVALHO, R.C.S.; VELOSO, A.V.; MARIN, D.B. Análise energética da produção de milho para silagem cultivado em diferentes espaçamentos. Energia na Agricultura, Botucatu, v.30, p.418428, 2015.

MONTANARI, R.; PANACHUKI, E.; LOVERA, L.H.; OLIVEIRA， I.S.; BONINI， C.S.B. Variabilidade espacial da produtividade de sorgo e de atributos físicos em um Planossolo. Revista Agro@mbiente, Boa Vista, v.7, n.3, p.252-261, 2013.

MONTANARI, R.; PANACHUKI, E.; LOVERA, L.H.; CORREA, A.R.; OLIVEIRA, I.S.; QUEIROZ, H.A.; TOMAZ, P.K. Variabilidade espacial da produtividade de sorgo e de atributos do solo na região do ecótono Cerrado-Pantanal, MS. Revista Brasileira de Ciência do Solo, Viçosa, v.39, n.2, p.385-396, 2015.

PIMENTEL, D. Handboock of energy utilization in agriculture.Boca Raton, Florida: CRC, 1980. 496p.

SANTOS, R.R.; SIMON, E.J. Análise energética do milho em sistema de plantio direto, no assentamento rural da fazenda Pirituba, Itaberá/SP. Energia na Agricultura, Botucatu, v.25, p.121$137,2010$.

REN, L.T.; LIU, Z.X.; WEI, T.Y.; XIE, G.H. Evaluation of energy input and output of sweet sorghum grown as a bioenergy crop on coastal saline-alkali land. Energy, v.47, p.166-173, 2012. 
RIQUETTI, N.B.; BENEZ, S.H.; SILVA, P.R.A. Demanda energética em diferentes manejos de solo e híbridos de milho. Energia na Agricultura, Botucatu, v.27, n.2, p.76-85, 2012.

RODRIGUES, J.G.L.; GAMERO, C.A.; NASCIMENTO, F.M.; FERNANDES, J.C. Demanda energética de máquinas agrícolas na implantação da cultura do sorgo forrageiro. EnergianaAgricultura, Botucatu, v.26, p.65-76, 2011

ROMANELLI, T.L.; MILAN, M. Energy balance methodology and modeling of supplementary forage production for cattle in Brazil.Scientia Agricola, Piracicaba, v.62, p.1-7, 2005.

SEFEEDPARI, P.; RAFIEE, S.; KOMLEH, S.H.P.; GHAHDEJARANI, M.A source-wise and operation-wise energy use analysis for corn silage production, a case study of Tehran province, Iran. International Journal of Sustainable Built Environment, n.1, p.158-166, 2013.

SERRA, P.; COLAUZZI, M.; AMADUCCI, S. Biomass sorghum production risk assessment analysis: A case study on electricity production in the Po Valley. Biomass and Bioenergy, n.96, p.7586, 2017.
SILVA, J.C.S.; WENDLING， B.; CAMARGO, R.; MENDONÇA, L.B.P.; FREITAS, M.C.M. Análise comparativa entre os sistemas de preparo do solo: aspectos técnicos e econômicos. EnciclopédiaBiosfera, Goiânia, v.7, n.12; p.1-11, 2011.

TAPHEE, G.B.; ONU, J.I.; ALAM, M.K.; JEN, E.I. Resource Use Efficiency in Sorghum Production in Lau Local Government Area, Taraba State, Nigeria. American Advanced Journal of Agricultural Science, v.1, n.1, p.18-24, 2015.

VELOSO, A.V.; CAMPOS, A.T.; PAULA, V.R.; DOURADO, D.C.; JÚNIOR, T.Y.; SILVA, E.B. Energetic efficiency of a deep bed swine production system. Engenharia Agrícola, Jaboticabal, v.32, n.6, p.1068-1079, 2012.

ZANINI, A.; CAMPOS, A.T.; PRESTES, T.V.M.; DALMOLIN, M.F.; CAMPOS, A.T.; KLOSOWSKI, E.S. Análise do consumo de energia na produção de silagem de milho em plantio direto. Acta Scientiarum, Maringa, v.25, p.249-253, 2003.

WOODS, J.; WILLIAMS, A.; JOHN K. HUGHES, J.K.; BLACK, M.; MURPHY, R. Energy and the food system. Philosophical Transactions of Royal Society, n.365, p.2991-3006, 2010. 